\title{
BLUE COMPACT GALAXIES AND THE PRIMORDIAL HELIUM ABUNDANCE DETERMINATION
}

\author{
Y.I. IZOTOV \\ Main Astronomical Observatory, Ukrainian National Academy of Sciences \\ Goloseevo, Kiev-22, 252650, Ukraine
}

AND

T.X. THUAN

Astronomy Department, University of Virginia

Charlottesville, VA 22903, USA

\begin{abstract}
We use a sample of $\mathbf{4 5}$ low-metallicity H II regions to determine the primordial helium abundance $Y_{p}$. We have carefully investigated the physical effects which may make the He I line intensities deviate from their recombination values such as collisional and fluorescent enhancements, underlying $\mathrm{He}$ I stellar absorption and absorption by Galactic interstellar $\mathrm{Na}$ I. By extrapolating the $Y$ vs. $\mathrm{O} / \mathrm{H}$ linear regression to $\mathrm{O} / \mathrm{H}=0$, we obtain $Y_{p}=0.245 \pm 0.002$. Our $Y_{p}$ gives $\Omega_{b} h_{50}^{2}=$ $0.06 \pm 0.01$.
\end{abstract}

\section{Introduction}

In the standard hot big bang model of nucleosynthesis (SBBN), four light isotopes, D, ${ }^{3} \mathrm{He},{ }^{4} \mathrm{He}$ and ${ }^{7} \mathrm{Li}$, were produced by nuclear reactions a few seconds after the birth of the Universe. Given the number of relativistic neutrino species and the neutron lifetime, the abundances of these light elements depend on one cosmological parameter only, the baryon-to-photon ratio $\eta$, which in turn is directly related to the density of ordinary baryonic matter $\Omega_{b}$. Thus precise abundance measurements of the four light elements can provide information about the mean density of ordinary matter in the Universe.

The primordial mass fraction $Y_{p}$ of ${ }^{4} \mathrm{He}$ is usually derived by extrapolating the $Y-\mathrm{O} / \mathrm{H}$ and $Y-\mathrm{N} / \mathrm{H}$ correlations to $\mathrm{O} / \mathrm{H}=\mathrm{N} / \mathrm{H}=0$ using various samples of dwarf irregular and blue compact galaxies (BCGs). These galaxies are the least chemically evolved galaxies known, so they contain very little helium manufactured by stars after the big bang. Because the $Y_{p}-\eta$ relation has a very small slope, $Y_{p}$ has to be determined with exquisite precision.

\section{Contamination by stellar absorption}

Several physical mechanisms discussed in Izotov et al. $(1994,1997)$ may make the He I line intensities deviate from their recombination values. While for the majority of BCGs, collisional and fluorescent enhancements of the He I line intensities are small, they can play an important role in some galaxies. The best way to take into account these effects is to use several of the brightest He I emission lines in the optical range ( $\lambda 3889, \lambda 4471, \lambda 5876, \lambda 6678$ and $\lambda 7065)$ and to solve self-consistently for the electron number density in the $\mathrm{He}^{+}$zone and the optical depth in the He I $\lambda 3889$ line to reproduce the theoretical He I line intensity recombination ratios.

Underlying He I stellar absorption can be important in some galaxies. Neglecting it can lead to misleadingly low helium mass fractions. A case in point is the NW component of $\mathrm{I} \mathrm{Zw} 18$ where the use of the He I $\lambda 6678$ line gives $Y=0.233 \pm 0.008$ and that of the He I $\lambda 4471$ line $Y \leq 0.200$, an unphysically low value. Izotov \& Thuan (1997) have argued that the NW component of I Zw 18 should not be used for $Y_{p}$ determination, but rather its $\mathrm{SE}$ component which is much less subject to 
underlying $\mathrm{He}$ I stellar absorption. The derived value for the SE component is considerably higher $Y=0.243 \pm 0.009$.

\section{Results and Discussion}

The results of the fits for the $Y-\mathrm{O} / \mathrm{H}$ and $Y-\mathrm{N} / \mathrm{H}$ linear regressions for our sample are given in Table 1. Our $Y_{p}$ gives $\Omega_{b} h_{50}^{2}=0.06 \pm 0.01$. In the framework of standard big bang nucleosynthesis theory, our derived $Y_{p}$ is consistent with a low value of the $\mathrm{D} / \mathrm{H}$ abundance as measured by Tytler et al. (1996) in absorption systems toward quasars, while it is inconsistent with the high $\mathrm{D} / \mathrm{H}$ abundance reported by Songaila et al. (1994).

TABLE 1. Maximum Likelihood Linear Regressions

\begin{tabular}{lcc}
\hline Method & Oxygen & Nitrogen \\
\hline All He I lines & $0.2451 \pm 0.0024+43 \pm 27(\mathrm{O} / \mathrm{H})$ & $0.2459 \pm 0.0017+882 \pm 429(\mathrm{~N} / \mathrm{H})$ \\
He I $\lambda 6678$ & $0.2433 \pm 0.0044+65 \pm 54(\mathrm{O} / \mathrm{H})$ & $0.2451 \pm 0.0031+1197 \pm 954(\mathrm{~N} / \mathrm{H})$ \\
\hline
\end{tabular}

\section{References}

Izotov, Y. I., Thuan, T. X., \& Lipovetsky, V. A. 1994, ApJ, 435, p. 647

Izotov, Y. I., Thuan, 'T. X., \& Lipovetsky, V. A. 1997, ApJS, 108, p. 1

Izotov, Y. I., \& Thuan, T. X. 1997, ApJ, in press

Songaila, A., Cowie, L. L., Hogan, C. J., \& Rugers, M. 1994, Nature, 368, p. 599

Tytler, D., Fan, X.-M., \& Burles, S. 1996, Nature, 381, p. 207 